\title{
Thermal energy storage by microcomposite of a phase change material and ethyl cellulose
}

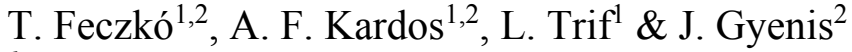 \\ ${ }^{1}$ Institute of Materials and Environmental Chemistry, \\ Research Centre for Natural Sciences, \\ Hungarian Academy of Sciences, Hungary \\ ${ }^{2}$ Research Institute of Chemical and Process Engineering, \\ Faculty of Information Technology, University of Pannonia, Hungary
}

\begin{abstract}
Phase change materials (PCMs) are capable of storing and releasing large amounts of latent heat thermal energy when undergoing phase change. They are developed for various building applications such as thermal energy storage, thermal protection, cooling, air-conditioning, waste heat recovery and for solar heating systems. Paraffin PCMs are low cost, have a moderate thermal energy density, but low thermal conductivity. PCM microencapsulation is one of the best tools to enhance the heat transfer rate by enlarging the surface area. In this work, ethyl cellulose as an environmental friendly encapsulating material was used to entrap n-hexadecane PCM by an emulsion-solvent evaporation method using poly(vinyl alcohol) (PVA), Tween 80 or poly(methacrylic acid sodium salt) (PMAA) emulsifier. The structure of the forming microparticles was predicted by determining the interfacial tensions between the phases. Both theoretically and in the experiments, composites prepared with PMAA showed the most desirable properties regarding the size (average: $80 \mu \mathrm{m}$ ) and the latent heat storage capacity (of melting and freezing were $111.4 \mathrm{~J} / \mathrm{g}$ and $117.9 \mathrm{~J} / \mathrm{g}$, respectively). Furthermore, there was no significant temperature and enthalpy change observed after 1000 heating-cooling thermal cycles. Thus, this microcomposite can be considered as suitable encapsulated PCM for thermal energy storage applications.
\end{abstract}

Keywords: phase change materials, latent heat storage, microencapsulation, ethyl cellulose. 


\section{Introduction}

Energy storage systems are essential for decreasing dependency on the fossil fuels and contributing to a more efficient environmental friendly energy use [3].

During sensible heating the thermal energy is stored up to the initiation of the melting process, whereas, the thermal energy stored during the phase change process is the latent heat. In solid state, the supplied heat to the PCM enhances its temperature until it reaches the melting point. The PCM temperature remains constant throughout the melting process. During the phase change process substantial amount of heat is stored. The latent heat storage employing PCM is the most effective way of the thermal energy storage. In a typical working temperature range, the latent heat storage capacity is about 5 to 14 times of the conventional thermal energy storage materials such as masonry, rock etc. [2]. Thus, the PCM provides much higher energy storage density with a smaller temperature oscillation, when compared with the sensible heat storage method. PCMs have received attention in solar heating system and building energy conservation. PCMs are developed for various applications due to their different melting-freezing temperature ranges: materials melting below $15^{\circ} \mathrm{C}$ are applied in air conditioning, while those ones changing phase above $90^{\circ} \mathrm{C}$ are used to drop the temperature if there is a sudden heat rising to prevent ignition. The materials that melt between these temperatures can be applied in solar heating and for heat load levelling [3]. So far, many PCMs such as salt hydrates, paraffins, fatty acids, fatty acid esters and their binary and ternary mixtures have been widely investigated for latent heat thermal energy storage due to their different phase change intervals. Paraffins have been widely used due to their high latent heat storage capacity and appropriate thermal properties, such as little or no supercooling, low vapor pressure, good thermal and chemical stability, and selfnucleating behavior.

However, most of the PCMs (especially organic PCMs) show a lower thermal conductivity, hence, results in a decreased heat transfer rate. The heat transfer can be enhanced by using fin configuration, inserting a high thermal conductivity metal matrix into a PCM, dispersing the PCM with particles of high thermal conductivity, or by micro-encapsulation of the PCMs.

Microencapsulation is a process of coating individual particles or droplets with a continuous film to produce capsules in micrometer to millimeter size. Microencapsulated phase change materials are composed of two main parts: a PCM as core and a polymer or inorganic shell as PCM container. The advantages of microencapsulated PCMs are the protection of PCM against the influences of the environment, the enhancement of specific heat-transfer area, and enabling the PCM core material to withstand changes in volume of the PCM, as the phase change occurs [2]. If the microcapsules are dispersed in a fluid such as water, they form a pumpable slurry that can be used as an energy storage medium, a socalled PCM slurry [4]. Thermal fluid, with microencapsulated PCM particles in it, possesses greater heat exchange ability owing to the excessive heat storage capacity. 
Recently, microencapsulation of PCMs have been provided interesting alternatives for utilizing PCMs in energy storage applications such as in thermal energy storage, thermal insulation, heating and air conditioning of buildings, as well as storage tanks and heat exchangers [5]. Ethyl cellulose (EC) is preferably used encapsulating compound mainly in the pharmaceutical technology e.g. for modifying drug release, further, nutraceutical, agricultural, dental, printing, clothing and cosmetic applications are also known.

Loxley and Vincent [6] studied the emulsion-solvent evaporation method most thoroughly for the entrapment of n-hexadecane in a poly(methylmethacrylate) (PMMA) shell. The morphology of microcapsules formed by solvent evaporation is determined by the interfacial energies to a high extent. The equilibrium morphology of forming drops in the presence of three immiscible liquids can be characterized considering interfacial tensions [7]. Briefly, if droplets of immiscible liquids (phases 1 and 3) contact with a third mutually immiscible liquid (phase 2), the final equilibrium morphology can be estimated after determining the interfacial tensions between the phases $\left(\gamma_{12}, \gamma_{23}\right.$, and $\left.\gamma_{13}\right)$. The spreading coefficients $S_{i}$ for each phase can be defined as

$$
\mathrm{S}_{\mathrm{i}}=\gamma_{\mathrm{jk}}-\left(\gamma_{\mathrm{ij}}+\gamma_{\mathrm{ik}}\right)
$$

and selecting phase 1 for which $\gamma_{12}>\gamma_{23}$, then $S_{1}<0$. At this boundary condition there are only three possible combinations of $\mathrm{S}_{\mathrm{i}}$, that is

$$
\begin{aligned}
& \mathrm{S}_{1}<0 ; \mathrm{S}_{2}<0 ; \mathrm{S}_{3}>0 \\
& \mathrm{~S}_{1}<0 ; \mathrm{S}_{2}<0 ; \mathrm{S}_{3}<0 \\
& \mathrm{~S}_{1}<0 ; \mathrm{S}_{2}>0 ; \mathrm{S}_{3}<0
\end{aligned}
$$

When the conditions in eqn (2) are satisfied, the particles possess a core-shell structure with a phase 1 core within a shell of phase 3 . When eqn (3) is accomplished, "acorn"-shaped particles form, and in the case of eqn (4), two separate droplets remain.

This approach was also applied by Loxley and Vincent [6] with the difference that relationship was given for one solid and two liquid phases. The structure of the formed microcapsules was predicted by analysing the interfacial tensions between the oil core and the various aqueous surfactants as well as between the encapsulating polymer and the emulsifier solutions.

In our study n-hexadecane PCM was microencapsulated into EC polymer by an emulsion-solvent evaporation method applying PVA, Tween 80 or PMAA emulsifiers. Size, morphology, yield, PCM content, energy storage capacity and thermal reliability of the forming composite particles were investigated.

\section{Materials and methods}

\subsection{Materials}

Dichloromethane, polyvinyl alcohol $\left(\mathrm{M}_{\mathrm{w}}=30,000-70,000,87-90 \%\right.$ hydrolysed $)$, Tween 80 , poly (methacrylic acid sodium salt) $\left(\mathrm{M}_{\mathrm{w}}=9500\right)$ were obtained from Sigma Aldrich, and n-hexadecane (HD) was purchased from MOL Hungarian 
Oil and Gas Company (Hungary). Ethyl cellulose (viscosity: $4 \mathrm{mPa} \mathrm{s}, 5 \% \mathrm{w} / \mathrm{w}$ in $80: 20$ toluene/ethyl alcohol, $25^{\circ} \mathrm{C}$ ) was a kind gift from Dow Deutschland Anlagengesellschaft mbH (Germany).

\subsection{Emulsion-solvent evaporation technique}

The phase change material was embedded by the EC polymer using an oil-inwater emulsion, solvent evaporation method. $400 \mathrm{mg}$ EC was dissolved in $5 \mathrm{ml}$ dichloromethane and $0.52 \mathrm{ml}(400 \mathrm{mg}) \mathrm{HD}$ was added during magnetic stirring. The oil-in-water emulsion was formulated by pouring the organic phase into $20 \mathrm{ml}$ distilled water containing $2 \% \mathrm{w} / \mathrm{v}$ PVA emulsifier, and the organic solvent was removed while magnetically stirred (IKA RET B) for 150 min with $700 \mathrm{rpm}$ under atmospheric pressure at room temperature. In another experiment the water phase consisted of $40 \mathrm{ml}$ solution of $1 \% \mathrm{w} / \mathrm{v}$ Tween 80 surfactant in distilled water with the same previous parameters. Finally, $400 \mathrm{mg}$ EC was dissolved in $5 \mathrm{ml}$ dichloromethane and $1.04 \mathrm{ml}(800 \mathrm{mg}) \mathrm{HD}$ was added during magnetic stirring. The oil-in-water emulsion was formulated by pouring the organic phase into $20 \mathrm{ml}$ distilled water containing 1\% w/v PMAA surfactant, and was stirred magnetically as given above. Composite microcapsules were filtered by a $3 \mu \mathrm{m}$ cut-off filter (Macherey-Nagel $640 \mathrm{~d}$ ) for the determination of yield and PCM content.

\subsection{Analysis}

The particle size distribution of microcapsules was determined by Mastersizer 2000 (Malvern Instruments, Malvern, UK) operated upon laser diffraction method. Their morphology was investigated after centrifuging and redispersion in distilled water, dropping onto a grid, and drying at room temperature. Then, the capsules were vacuum-coated for $3 \mathrm{~min}$ with a mixture of gold and palladium and examined with a Philips XL-30 environmental scanning electron microscope (ESEM) at $25 \mathrm{kV}$.

The yield of the product was determined by weighing the microcapsules after filtration, rinsing with distilled water and drying at $60^{\circ} \mathrm{C} .100 \mathrm{mg}$ of each sample was weighed, and the PCM content was calculated after the HD was extracted 3 times from the microparticles by each $5 \mathrm{ml} \mathrm{n}$-hexane.

The thermal properties of the microcapsules were determined using a Setaram $\mu \mathrm{DSC} 3$ evo differential scanning microcalorimeter. The samples were weighed into $100 \mu \mathrm{L}$ aluminium crucibles, and were gently pressed to the bottom of the crucible in order to improve the thermal contact between the sample and the crucible, then, the system was hermetically closed by crimping a cap. The samples were three times cycled from 5 to $35^{\circ} \mathrm{C}$, with a scanning rate of $0.6^{\circ} \mathrm{C} / \mathrm{min}$. The results were processed by using the thermoanalyzer's Calisto Processing (v1.12) software. The corresponding melting/freezing enthalpies, and onset temperatures were determined by the baseline integration method (tangential sigmoid baseline type).

The thermal reliability of the microencapsulated PCM was determined in accelerated thermal cycling tests. One thermal cycling test consisted of exposing 
microcapsules to a melting and freezing process. The microcapsules were warmed by a heating circulator (Julabo EH, Germany) up to $29^{\circ} \mathrm{C}$ and cooled down to $9^{\circ} \mathrm{C}$ by a Peltier element consecutively up to 1000 thermal cycles. The thermal storage properties of the treated microcapsules were also investigated by differential scanning calorimetry (DSC) in order to check their thermal reliability.

\subsection{Determination of interfacial tensions and spreading coefficients}

Polymer films were prepared from an $8 \%$ and a $4 \%(\mathrm{w} / \mathrm{v})$ solution of the ethyl cellulose polymer in DCM. A glass microscope slide, previously cleaned with ethanol, was immersed in the polymer solution. It was wetted by the solution and after five minutes the solution was drained by $1 \mathrm{~cm} / \mathrm{min}$. Finally, the freshly prepared film was dried overnight.

The surface tensions of the liquid phase components of the systems were measured by the pendant drop method using an FTA 1000 B tensiometer (First Ten Angstroms, Inc., USA). The interfacial tensions between HD and the surfactant solutions were also determined by the same method using a quartz cuvette filled with the oil (light phase). A drop of the surfactant solution was pictured when immersed in HD in a quartz cuvette [8]. The interfacial tensions between each surfactant solution, oil and ethyl cellulose polymer were calculated from the measured contact angle against a film of ethyl cellulose on the base of Young's Force Balance for the sessile drop.

$$
\gamma_{\mathrm{LV}} \cdot \cos (\theta)=\gamma_{\mathrm{SV}}-\gamma_{\mathrm{SL}}
$$

where LV: liquid - vapour interface, SV: solid (polymer) - vapour interface, SL: solid - liquid interface and $\theta$ : contact angle between polymer film and liquid phase.

The surface tension of the ethyl cellulose polymer $(32 \mathrm{mN} / \mathrm{m})$ was taken from the pertinent literature [9]. Interfacial tension values were determined from at least ten independently formed drops. The temperature of experiments was $22 \pm$ $1{ }^{\circ} \mathrm{C}$.

\section{Results and discussion}

\subsection{Microcapsules size, yield and PCM content}

All the three emulsifiers (PVA, Tween 80 and PMAA) were suitable to form composite microcapsules, nevertheless, the emulsifiers influenced the size of microcapsules substantially. The forming microcapsules were found to be the largest with Tween 80 (volume weighted mean: $342 \mu \mathrm{m}$ ), and PVA (volume weighted mean: $92 \mu \mathrm{m}$ ) or PMAA (volume weighted mean: $80 \mu \mathrm{m}$ ) resulted in smaller, more desirable particles (Figure 1). PVA and PMAA were similarly effective surface active agents, nevertheless, microparticles manufactured by PMAA had a wider size distribution. 


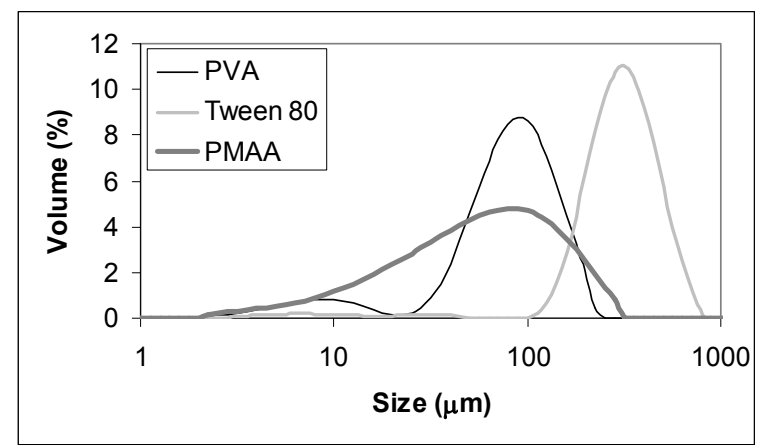

Figure 1: Size distribution of the EC-HD composites manufactured by PVA, Tween 80 and PMAA emulsifiers.

The yield of the microparticles was moderately high with PVA and Tween 80 (Table 1), while PMAA provided very satisfactory results. Initial HD content was selected to be the highest $(66.7 \%)$ during the preparation using PMAA, since significant leakage could be observed at a higher initial HD ratio utilizing the other two emulsifiers. The HD content in the microcapsules formed by PVA or PMAA was very close to its initial weighed ratio $(50 \%$ and $66.7 \%$, respectively); nevertheless with Tween 80 it was significantly lower (initial ratio: $50 \%$ ). This supports the fact found also in size measurements that the Tween 80 was not as efficient emulsifier as PMAA or PVA.

Table 1: $\quad$ Yield (\%) and PCM content (\%) of ethyl cellulose-n-hexadecane microcapsules prepared by PVA, Tween 80 and PMAA surfactants.

\begin{tabular}{|c|c|c|}
\hline Type of emulsifier & Yield (\%) & PCM content (\%) \\
\hline PVA & 82.1 & 49.7 \\
\hline Tween 80 & 94.0 & 41.3 \\
\hline PMAA & 95.0 & 63.6 \\
\hline
\end{tabular}

\subsection{Microcapsule morphology}

As SEM images (Figure 2) show, microcapsules were spherical prepared by either of the emulsifiers. Scanning electron microscopy also indiced that PMAA emulsifier was appropriate to produce real core-shell microcapsules with compact shell (Figure 2E), F)). Shell of microcapsules formed by Tween 80 represented very high porosity, and some of the pores seemed to perforate the shell (Figure 2C), D)), which can result in the leakage of the PCM from the core. The wall of microparticles synthesized by PVA was also porous, though much smaller holes can be observed on the shell of some microspheres (Figure 2A), B)), they might allow the PCM to leak out. 
A)

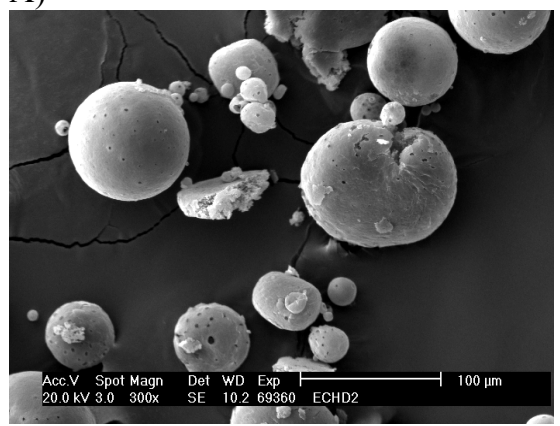

C)

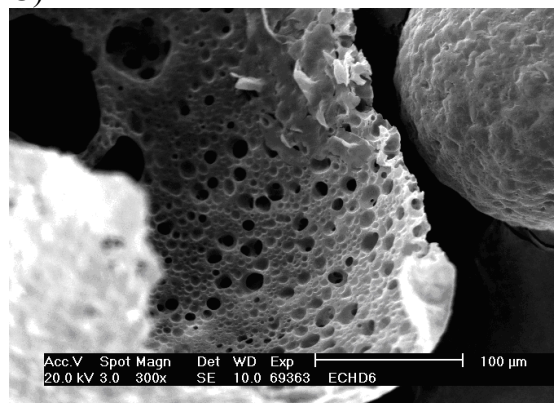

E)

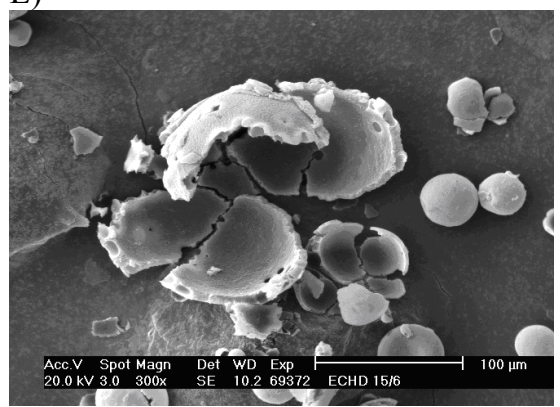

B)

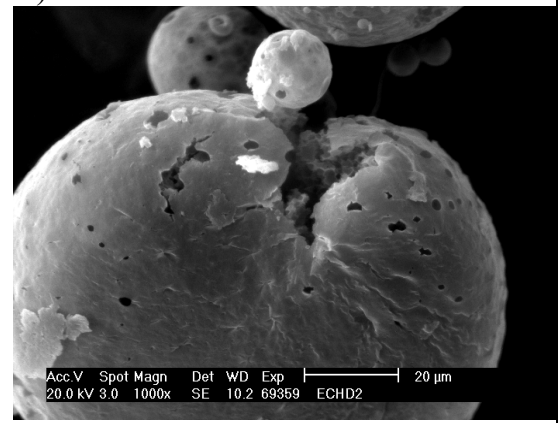

D)

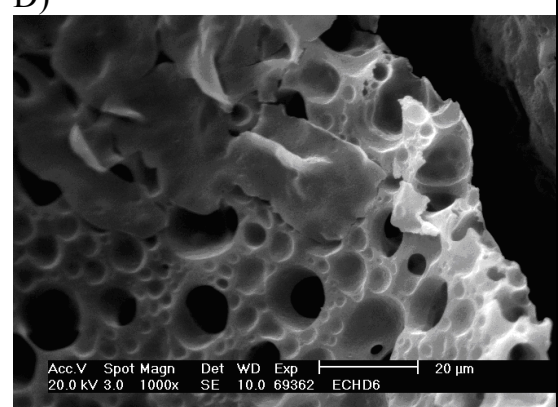

F)

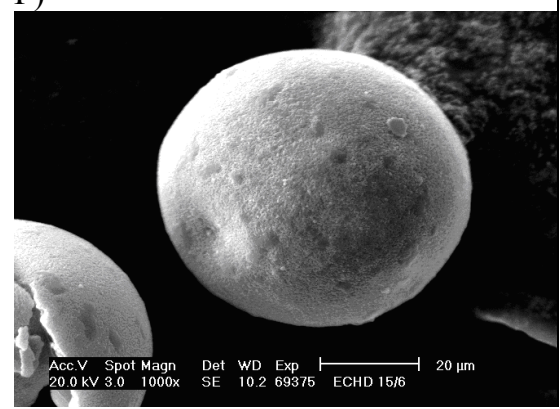

Figure 2: $\quad$ SEM images of EC-HD microcapsules prepared by PVA (A, B), Tween 80 (C, D, E) and PMAA (F, G).

\subsection{Estimation of morphology}

Via application of the model created by Torza and Mason [7] for our system, the core-shell morphology can be predicted, if the conditions in eqn (2) are fulfilled. The interfacial tensions between each emulsifier solution and the air, as well as between the HD and each surfactant solution had to be measured in order to determine the equilibrium morphology. Table 2 shows the measured surface tensions $(\mathrm{mN} / \mathrm{m})$ (liquid-vapour of surfactant solution and HD, liquid-liquid of surfactant solution and HD). Table 3 presents contact angle (degrees) data (EC 
film - surfactant solution and EC film - HD), calculated interfacial tensions and spreading coefficients (solid-liquid of EC film - surfactant solution and that of $\mathrm{EC}$ film - HD).

Table 2: $\quad$ Measured surface tension $(\mathrm{mN} / \mathrm{m})$ values (L-liquid, $\mathrm{V}$-vapour).

\begin{tabular}{|c|c|c|c|c|c|c|}
\hline No. & $\begin{array}{c}\text { Surfactant, } \\
\text { conc. } \\
(\% \mathrm{w} / \mathrm{v})\end{array}$ & $\begin{array}{c}\text { Polymer, } \\
\text { conc. } \\
(\% \mathrm{w} / \mathrm{v})\end{array}$ & PCM & $\begin{array}{c}\text { Surfactant } \\
\text { solution L-V }\end{array}$ & $\begin{array}{c}\text { HD } \\
\text { L-V }\end{array}$ & $\begin{array}{c}\text { Surfactant } \\
\text { solution-HD } \\
\text { L-L }\end{array}$ \\
\hline 1 & Tween, $1 \%$ & $4 \%$ & HD & 46.16 & 26.94 & 11.73 \\
\hline 2 & PVA, $\%$ & $8 \%$ & HD & 46.82 & 26.94 & 16.29 \\
\hline 3 & PMAA, 1\% & $8 \%$ & HD & 73.34 & 26.94 & 32.51 \\
\hline
\end{tabular}

Table 3: Contact angles (degrees), calculated interfacial tensions and spreading coefficients (S-solid, L-liquid).

\begin{tabular}{|c|c|c|c|c|c|c|c|}
\hline \multirow{2}{*}{ No. } & \multicolumn{3}{|c|}{$\begin{array}{c}\text { Surfactant solution-EC film S-L, } \\
\text { HD-EC film S-L }\end{array}$} & \multirow{2}{*}{ S3 } & S2 & S1 \\
\cline { 2 - 5 } & contact angle & \multicolumn{2}{|c|}{ interfacial tension } & & & \\
\hline 1 & 72.12 & 21.48 & 17.83 & 6.93 & -13.03 & -22.63 & -0.83 \\
\hline 2 & 72.84 & 20.39 & 18.19 & 6.75 & -8.64 & -27.73 & -4.85 \\
\hline 3 & 66.56 & 20.39 & 2.83 & 6.75 & 22.94 & -28.59 & -36.4 \\
\hline
\end{tabular}

Spreading coefficients vary in quite a wide range as a function of applied emulsifiers. As expected, when the HD-surfactant liquid-liquid interfacial tension was the smallest with Tween 80 , the spreading coefficient $\mathrm{S}_{3}$ was the highest negative value. When the liquid-liquid interfacial tension was the biggest with PMAA, the $\mathrm{S}_{3}$ became positive. This means core-shell structure is most likely to be formed using PMAA, while acorn shape is probable with Tween 80 and PVA. In our investigations, the morphologies obtained by SEM showed quasi core-shell structure with Tween 80 and PVA, which means that a core could be observed inside the polymer shell, but the porousness of the wall must issue in the leakage of oil. In the initially forming emulsion droplets the solubility of ethyl cellulose decreases via the evaporation of dichloromethane. Thus, in the emulsion droplets ethyl cellulose-rich coacervates form, which move to the surface and coalesce, although, due to the wetting condition they cannot constitute a completely continuous shell in the presence of Tween 80 or PVA. The pores of the shell are filled by the droplets of HD, though the energetic relations do not enable their movement into the water phase, hence they remain in the EC shell as droplets. At the same time, the dichloromethane evaporates from the emulsion, which issues in increasing viscosity and mechanical strength of the shell material. This impedes the leakage of HD in bigger drops to the outward capsule surface. However, using Tween 80 provided such big holes in the shell that makes probable significant leakage of the PCM. 


\subsection{Heat energy storage of microcapsules}

DSC investigations were carried out to quantify the energy storage capacity of microcapsules. Structure of microcapsules formulated with PVA and especially with PMAA denoted potential applicability for efficient and durable PCM entrapment. The DSC curve of HD (not shown) was taken as reference to evaluate the change in the thermal properties of the composite PCM (Figure 3).

A)

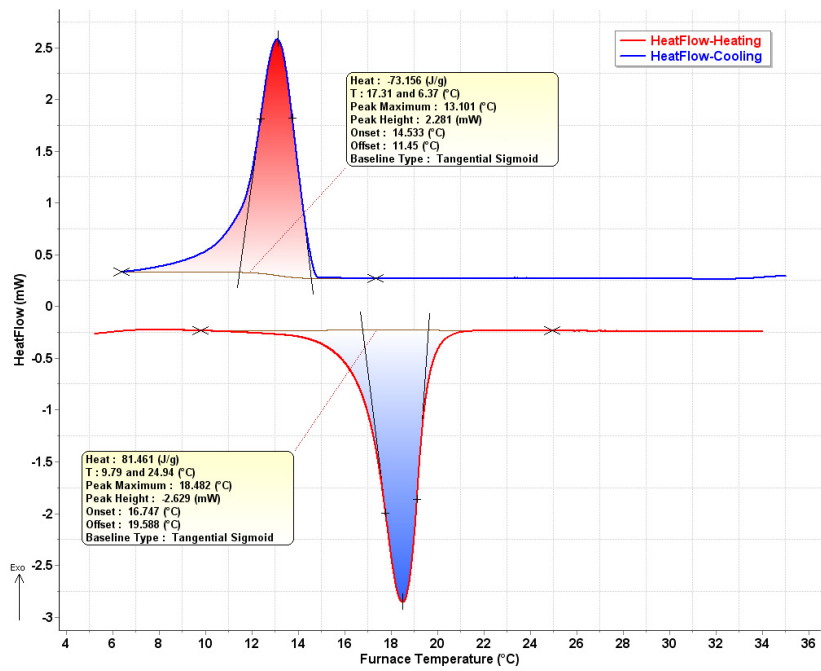

B)

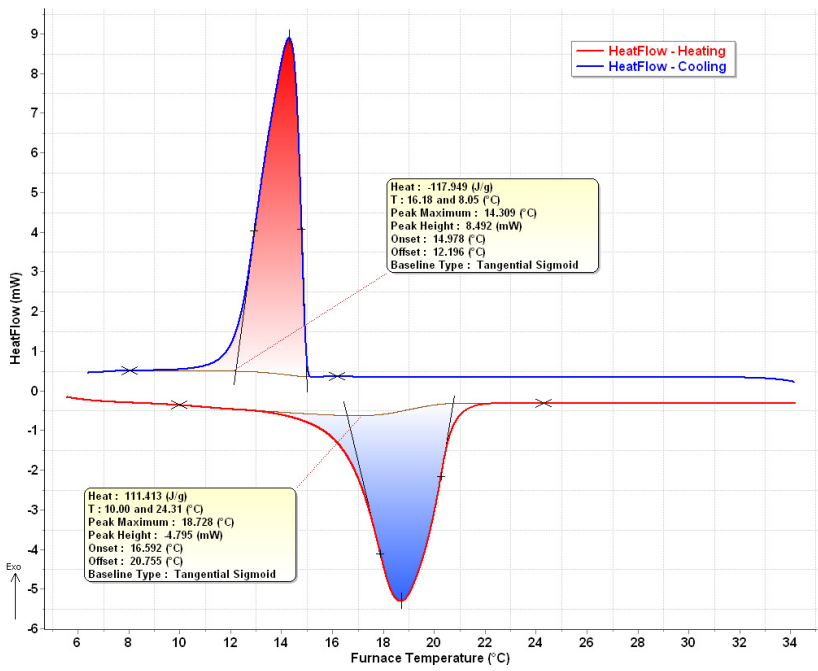

Figure 3: DSC of ethyl cellulose-n-hexadecane composites manufactured by PVA A) and PMAA B) surfactants. 
The phase transition intervals of the EC-HD microcapsules prepared with PVA and PMAA greatly overlapped with those of the HD, suggesting that the thermal properties of the composites were similar to those of the HD.

The melting and solidification temperatures are $18.9^{\circ} \mathrm{C}$ and $14.9^{\circ} \mathrm{C}$ for pure $\mathrm{HD}, 18.5^{\circ} \mathrm{C}$ and $13.1^{\circ} \mathrm{C}$ for the EC-HD by PVA, and $18.7^{\circ} \mathrm{C}$ and $14.3^{\circ} \mathrm{C}$ for the EC-HD by PMAA, respectively. When the phase transition temperatures of PCM and microcapsules synthesized by PVA and PMAA are taken into consideration comparatively, the melting temperatures are in compliance with the HD. This is due to the ordered structures of PCM in the solid phase gradually changed towards the disordered structures in the liquid phase of HD [1]. Conversely, the entrapped impurities (e.g. trace of emulsifier) and the heterogeneous structure reduced the crystallization point [10].

The latent heats of melting and freezing were $208.4 \mathrm{~J} / \mathrm{g}$ and $206.6 \mathrm{~J} / \mathrm{g}$ for HD, $111.4 \mathrm{~J} / \mathrm{g}$ and $117.9 \mathrm{~J} / \mathrm{g}$ for the EC-HD microcapsules by PMAA, $81.5 \mathrm{~J} / \mathrm{g}$ and $73.2 \mathrm{~J} / \mathrm{g}$ for the EC-HD composite by PVA, respectively. The thermal energy storage capacity of particles prepared by PMAA was naturally substantially higher because of the higher PCM content, however, with PVA using higher PCM content than 50\% represented significant amount of PCM on the surface of the capsules.

The PCMs designed for application must withstand repetitive cycles of heating and cooling without any degradation in their life period. Results of the DSC analysis after the thermal reliability study can be seen in Figure 4. In accordance with expectations, the microcapsules predicted and observed to have core-shell structure (by PMAA) could save their energy storing capacity almost completely (enthalpies: $108.5 \mathrm{~J} / \mathrm{g}$ and $115.3 \mathrm{~J} / \mathrm{g}$ ). The decrease of latent heats of melting and freezing of composites formed by PVA was considerable, $70.5 \mathrm{~J} / \mathrm{g}$ and $68.1 \mathrm{~J} / \mathrm{g}$, respectively.

\section{Conclusions}

Ethyl cellulose was found to be a suitable polymer to entrap n-hexadecane phase change material. Microcapsules were encapsulated by the emulsion-solvent evaporation method using PVA, Tween 80 or PMAA emulsifier. The structure of the forming microcapsules was predicted by determining the interfacial tensions between the phases, and analysing the spreading coefficients. In accordance with the theoretical calculations, composites prepared with PMAA showed the most desirable properties regarding the size and the latent heat storage capacity; furthermore, there was no significant temperature and enthalpy change observed after thermal cycling. Due to its simple preparation method, good thermal energy storage capacity and thermal reliability, this microcomposite can be considered as suitable encapsulated PCM for thermal energy storage applications. 
A)

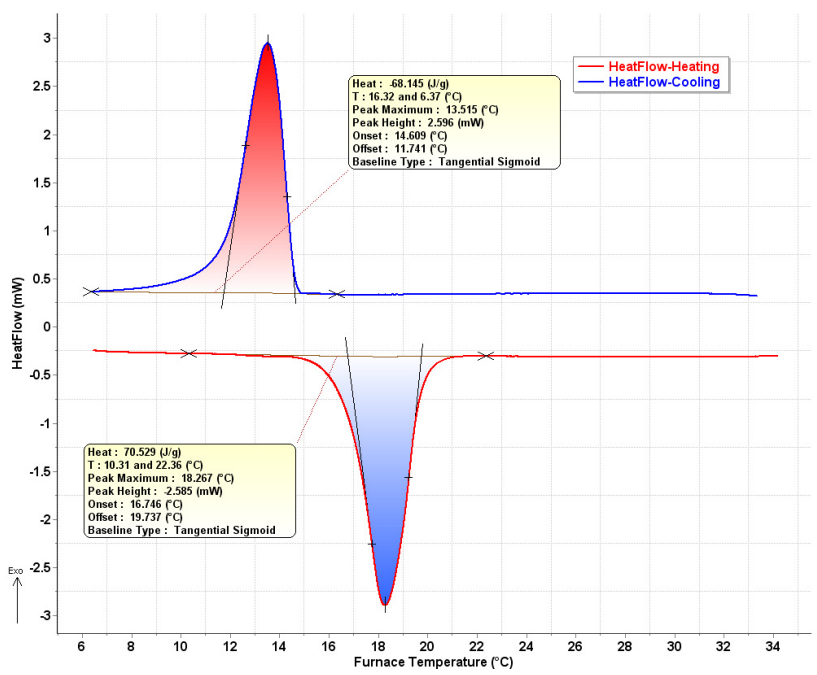

B)

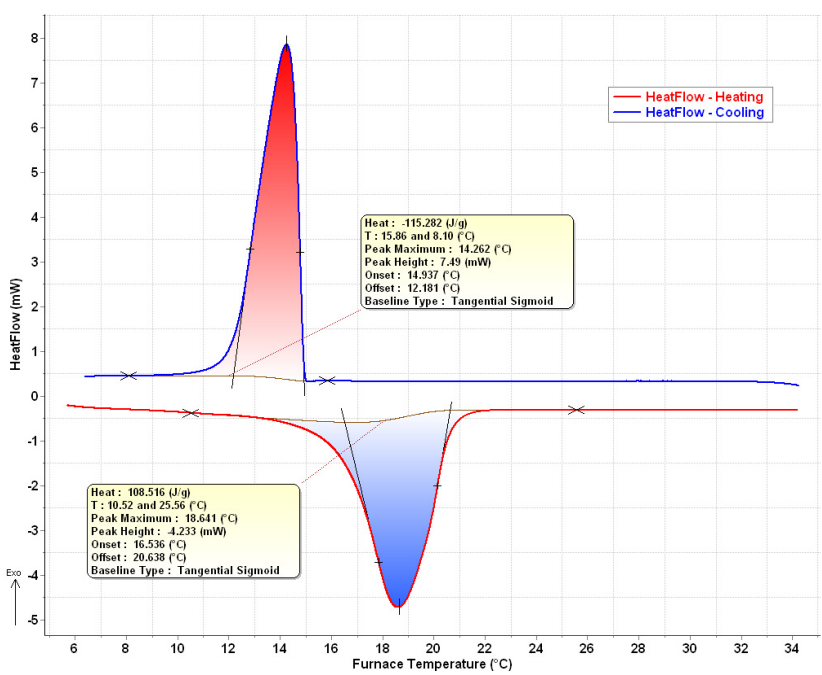

Figure 4: DSC of ethyl cellulose-n-hexadecane composites manufactured by using PVA A) and PMAA B) emulsifiers after 1000 thermal cycles.

\section{Acknowledgements}

We acknowledge the support of the Hungarian state and the European Union TAMOP-4.2.2A-11/1/KONV-0443560/130 and TAMOP-4.2.1-08/1-2008-0007. Ethyl cellulose supply is courtesy of Dow Deutschland Anlagengesellschaft 
$\mathrm{mbH}$. We are grateful to Dr. Gyula Kiss for supplying the tensiometer for the measurements.

\section{References}

[1] Zhang, T., Wang, Y., Shi, H. \& Yang, W., Fabrication and performances of new kind microencapsulated phase change material based on stearic acid core and polycarbonate shell. Energy Conversion and Management, 64, pp. 1-7, 2012.

[2] Salunkhe, P.B. \& Shembekar, P.S., A review on effect of phase change material encapsulation on the thermal performance of a system. Renewable and Sustainable Energy Reviews, 16, pp. 5603-5616, 2012.

[3] Fang, G., Li, H., Chen, Z. \& Liu, X., Preparation and characterization of flame retardant $n$-hexadecane/silicon dioxide composites as thermal energy storage materials. Journal of Hazardous Materials, 181, pp. 10041009, 2010.

[4] Heinz, A. \& Streicher, W., Application of phase change materials and PCM slurries for thermal energy storage, Proc. of the Ecostock Conference, Pomona, USA, 8B-4, 2006.

[5] Sarı, A., Alkan, C., Karaipekli, A. \& Uzun, O., Microencapsulated noctacosane as phase change material for thermal energy storage. Solar Energy, 83, pp. 1757-1763, 2009.

[6] Loxley, A. \& Vincent, B., Preparation of poly(methylmethacrylate) microcapsules with liquid cores. Journal of Colloid and Interface Science, 208, pp. 49-62, 1998.

[7] Torza, S. \& Mason, S.G., Three-phase interactions in shear and electrical fields. Journal of Colloid and Interface Science, 33, pp. 67-83, 1970.

[8] Luz, J.A.M., Soares, A., Interfacial Tension from Profile of the Pendant Drop Versus du Noüy's Method. XIII. International Mineral Processing Symposium, E-Book of Abstracts, eds. H. Özdag, V. Bozkurt, H. Ipek \& K. Bilir, Bodrum, Turkey, pp. 297-303, 2012.

[9] Brandrup, J., Immergut \& E.H., Grulke, E.A., Polymer Handbook, Fourth Edition, Wiley, VI, p. 533, 2003.

[10] Sarier, N. \& Onder, E., The manufacture of microencapsulated phase change materials suitable for the design of thermally enhanced fabrics. Thermochimica Acta, 452, pp. 149-160, 2007. 